\title{
A SYMMETRIZABLE SPACE THAT IS NOT PERFECT
}

\author{
DENNIS A. BONNETT ${ }^{1}$
}

\begin{abstract}
An example is given of a Hausdorff symmetrizable space which has a closed subset that is not a $G_{\delta}$-subset (thus, it is not perfect) and which is not subparacompact. This example is then used in the construction of a symmetrizable $T_{1}$-space $Y$ having a point $x_{0}$ such that $\left\{x_{0}\right\}$ is not a $G_{\delta}$-subset of $Y$.
\end{abstract}

A topological space $S$ is said to be a symmetrizable space provided that there is a distance function (or "symmetric") $d$ defined for $S$ such that, if each of $x$ and $y$ is a point of $S$, then (1) $d(x, y)=d(y, x),(2) d(x, y) \geqq 0$ and $d(x, y)=0$ if and only if $x=y$, and (3) a subset $P$ of $S$ is closed if and only if $d(x, P)>0$ for each $x$ in $S-P$. The space $S$ is said to be semimetrizable if $d$ satisfies conditions (1) and (2), and if condicion (3) is replaced by ( $\left.3^{\prime}\right)$ a point $p$ is a limit point of a subset $M$ of $S$ if and only if $d(p, M)=0$. A topological space is said to be perfect if every closed subset of $S$ is a $G_{\delta}$-subset. A topological space $S$ is said to be subparacompact if every open cover of $S$ has a $\sigma$-discrete closed refinement.

The symmetrizable spaces were defined by A. V. Arhangel'skiì, in [1], and a great deal of work has been done in investigating the properties of such spaces and in determining their relationship to other classes of abstract topological spaces (see [2], [5], and [8]). In [8], S. Nedev makes the inquiry, attributed to E. Michael, as to whether or not every symmetrizable space is perfect. This question becomes especially interesting in view of the fact that the closely related semimetrizable spaces are perfect. In this paper an example will be given of a Hausdorff symmetrizable space that is not perfect. In addition, it will be shown that this space is not subparacompact, answering an inquiry of D. K. Burke, in [4], as to whether or not each symmetrizable space is subparacompact. It should be noted that the space of Example 1 is not regular. Thus, it remains an open question whether each regular symmetrizable space is perfect (or subparacompact).

Received by the editors October $11,1971$.

AMS 1970 subject classifications. Primary 54A05, 54E25; Secondary 54E20, 54G20.

Key words and phrases. Abstract spaces, symmetrizable, semimetrizable, $G_{\delta}$-subset, subparacompact, perfect space.

1 This paper is part of the author's doctoral dissertation written under the direction of Professor R. W. Heath at Arizona State University.

(c) American Mathematical Society 1972 
ExAmple 1. Let $S$ denote the set of points in the coordinate plane topologized by specifying a subset $U$ of $S$ to be open if and only if the intersection of $U$ with any vertical or horizontal line is open relative to the Euclidean topology of that line. Define a distance function for $S$ by $d(z, w)=|z-w|$, if $z$ and $w$ are on the same vertical or horizontal line $(|z-w|$ indicates the Euclidean distance between $z$ and $w)$, and $d(z, w)=1$ otherwise.

It is clear that the distance function $d$ is a symmetric for the topology of $S$ and that the topology of $S$ contains the Euclidean topology of the plane. The space $S$ has been considered previously in the literature by J. Novak, in [9], and later by A. V. Arhangel'skiř, in [3]. It will be shown that the space $S$ is not perfect. Hereafter, $S$ will always denote the space of Example 1.

Lemma 2. There exists a subset $G$, of $S$, which is closed in the space $S$, dense in the coordinate plane relative to the Euclidean topology, and consists of exactly one point from each vertical and each horizontal line in $S$.

Proof. The set $G$ is the graph of a discontinuous real valued function $f$, of a real variable, such that $f(x+y)=f(x)+f(y)$ for all $x$ and $y$ in $R$. A proof of the existence of such a function (including the Euclidean denseness of its graph) has been done by G. Hamel, in [6].

LeMma 3. If $H$ is an open subset of $S$ containing the set $G$ of Lemma 2 and if $l_{x}=\{(x, y) \mid y$ is in $R\}$, then $A=\left\{x\right.$ in $R \mid l_{x} \cap(S-H)$ is second category in $\left.l_{x}\right\}$ is first category in $R$.

Proof. Assume that $A$ is second category in $R$. Since $S-H$ is closed in $S$, it follows that for each $x$ in $A$, the set $l_{x} \cap(S-H)$ is closed relative to the Euclidean topology on $l_{x}$. Thus, for each $x$ in $A$, the set $l_{x} \cap(S-H)$ contains a closed interval $I_{x}$. It will be shown that there exists a natural number $n$ and a second category (in the reals) subset $B$ of $A$, such that for each $x$ in $B$, the closed interval $I_{x}$ has length at least $1 / n$. Assume that no such $n$ and $B$ exists. Then, for each $n$ in $N$, the set $A_{n}=\{x \mid x$ is in $A$ and the length of $I_{x}$ is equal to $\left.1 / n\right\}$ is of first category in $R$. Therefore, $A=\bigcup\left\{A_{n} \mid n=1,2,3, \cdots\right\}$ is of first category in $R$ and a contradiction is obtained.

Let $n, A$, and $B$ be as described above. Define $D=\left\{D_{i} \mid i=1,2,3, \cdots\right\}$ where

$$
\begin{aligned}
D_{i}=\left\{(x, y) \mid \frac{i-1}{4 n} \leqq y \leqq \frac{i+1}{4 n} \text { and } x \text { is in } R\right\}, & \begin{array}{l}
\text { for } i \text { an odd } \\
\text { natural number, and }
\end{array} \\
=\left\{(x, y) \mid-\frac{i}{4 n} \leqq y \leqq \frac{2-i}{4 n} \text { and } x \text { is in } R\right\}, & \begin{array}{l}
\text { for } i \text { an even } \\
\text { natural number. }
\end{array}
\end{aligned}
$$


It will be shown that there exists a natural number $m$ and a second category (in the reals) subset $C$ of $B$, such that for each $x$ in $C,\left(I_{x} \cap D_{m}\right)=$ $\left(l_{x} \cap D_{m}\right)$. Assume that no such $m$ exists. Then for each $m$, the set $B_{i}=$ $\left\{x \mid x\right.$ is in $B$ and $\left.\left(I_{x} \cap D_{i}\right)=\left(l_{x} \cap D_{i}\right)\right\}$ is first category. But, for each $x$ in $B$, the length of $I_{x}$ is at least $1 / n$ and the length of $l_{x} \cap D_{i}$ is $1 / 2 n$, for $i=$ $1,2, \cdots$. Therefore, $B=\bigcup\left\{B_{i} \mid i=1,2,3, \cdots\right\}$ and consequently, $B$ is first category in $R$ and a contradiction is obtained.

Let $n, A, B, m$, and $C$ be as described in the previous paragraphs. Since $C$ is second category in $R$, it must be dense in some closed interval $I$ in $R$. Let $K=\bigcup\left\{I_{x} \mid x\right.$ is in $\left.C\right\}$. The set $K$ is Euclidean dense in a rectangle $K^{\prime}$, having width equal to the length of the closed interval $I$ and having height $1 / 2 \mathrm{~m}$. Since $G$ is a Euclidean dense subset of $S$, it follows that some point $p$, of $G$, is in $K^{\prime}$. But this implies that $d(p, K)=0$, and thus $p$ cannot be an interior point of $H$. This is a contradiction. Therefore, $A$ is first category in $R$.

\section{THEOREM 4. The symmetrizable space $S$ is not perfect.}

Proof. It will be shown that the set $G$ of Lemma 1 is not a $G_{\delta}$-subset of $S$. Assume that $G=\bigcap\left\{G_{i} \mid i=1,2,3, \cdots\right\}$ where each $G_{i}$ is open in $S$. Since each $G_{i}$ is an open set containing $G$, the set $A_{i}=\{x \mid x$ is in $R$ and $l_{x} \cap\left(S-G_{i}\right)$ is second category in $\left.l_{x}\right\}$ is first category in $R$ by Lemma 3 . Let $A=R-\bigcup\left\{A_{i} \mid i=1,2,3, \cdots\right\}$. Then $A$ is second category in $F$. If $x$ is in $A$, then for each $i, l_{x} \cap\left(S-G_{i}\right)$ is first category in $l_{x}$. Thus, $l_{x} \cap(S-G)=$ $\bigcup\left\{l_{x} \cap\left(S-G_{i}\right) \mid i=1,2, \cdots\right\}$ is first category in $l_{x}$. Therefore, for each $x$ in $A, l_{x} \cap G$ is second category, which contradicts $G$ containing but one point from each vertical and each horizontal line. Thus, $G$ is not a $G_{\delta^{-}}$ subset of $S$.

THEOREM 5. The symmetrizable space $S$ is not subparacompact.

Proof. Assume that the space $S$ is subparacompact. Let $G$ be the closed subset of $S$ defined in Lemma 2. Define $U_{x}=S-(G-\{x\})$. Then, for each $x$ in $G$, the set $U_{x}$ is open and contains only one point of $G$. Define $U=\left\{U_{x} \mid x\right.$ is in $\left.G\right\}$. Since $U$ is an open cover of $S$, there is a refinement $\mathscr{D}=\bigcup\left\{\mathscr{D}_{i} \mid i=1,2,3, \cdots\right\}$ of $U$, such that for each $i, \mathscr{D}_{i}$ is a discrete collection of closed sets. For each $i$ in $N$, let $F_{i}=\bigcup\left\{D\right.$ in $\left.\mathscr{D}_{i} \mid D \cap G=\Phi\right\}$. Since for each $i, F_{i}$ is the union of a subcollection of a discrete collection of closed sets, it follows that $F_{i}$ is closed. Since the Euclidean topology of the plane is a subset of the topology of $S$, each point of $S$ is a $G_{\delta^{-}}$ subset. Thus, for each $x$ in $S$, there exists a sequence of open sets $A_{1 x}$, $A_{2 x}, A_{3 x}, \cdots$ such that $\{x\}=\bigcap\left\{A_{i x} \mid i=1,2,3, \cdots\right\}$. For each $i$ in $N$, it is clear that each point $x$ in $G$ is contained in at most one member of $\mathscr{D}_{i}$. For each $i$, let $G_{i}=\left\{x\right.$ in $G \mid x$ is in some member of $\left.\mathscr{D}_{i}\right\}$. If $x$ is in $G_{i}$, 
let $D_{i x}$ be the member of $\mathscr{D}_{i}$ such that $x$ is in $D_{i x}$. For each $i$ and $j=$ $1,2,3, \cdots$, let $K_{i}(j)=\bigcup\left\{D_{i x} \cap\left(S-A_{j x}\right) \mid x\right.$ is in $\left.G_{i}\right\}$. Since each $K_{i}(j)$ is the union of a subcollection of a discrete collection of a closed sets, it follows that $K_{i}(j)$ is closed for each $i$ and $j$.

Thus $S-G=\left(\bigcup\left\{F_{i} \mid i=1,2,3, \cdots\right\}\right) \cup\left(\bigcup\left\{K_{i}(j) \mid i, j=1,2,3, \cdots\right\}\right)$. It has been shown that $S-G$ is an $F_{\sigma}$-subset of $S$. Therefore, $G$ must be a $G_{\delta}$-subset. This contradicts Theorem 4 . It follows that the space $S$ is not subparacompact.

The space $S$ will now be used in the construction of a $T_{1}$-symmetrizable space $Y$ having a point $x_{0}$ such that $\left\{x_{0}\right\}$ is not a $G_{\delta}$-subset of $Y$.

THEOREM 6. There is a symmetrizable space containing a point which is not a $G_{\delta}$-subset.

Proof. Let $S$ be the space of Example 1, $d$ the symmetric for $S, G$ the subset of $S$ described in Lemma 2, and let $x_{0}$ be a point of $G$. Let $y=(S-G) \cup\left\{x_{0}\right\}$, and define a function $f$ from $S$ onto $Y$ by

$$
\begin{aligned}
f(x)=x, & \text { if } x \text { is in } S-G \text { and } \\
=x_{0}, & \text { if } x \text { is in } G .
\end{aligned}
$$

Let $d^{\prime}$ be a distance function for $Y$ defined by

$$
\begin{aligned}
& d^{\prime}(x, y)=d(x, y) \text { if } x \text { and } y \text { are not equal to } x_{0}, \\
& =d(x, G) \text { if } y=x_{0} \text { and } \\
& =d(y, G) \text { if } x=x_{0} \text {. }
\end{aligned}
$$

Topologize $Y$ by defining a subset $P$ of $Y$ to be closed if and only if $d^{\prime}(x, P)>0$ for any $x$ in $Y-P$.

It is a routine exercise to show that $f$ is a continuous function from the space $S$ onto the symmetric space $Y$. The set $\left\{x_{0}\right\}$ is not a $G_{\delta}$-subset of $Y$, since $G$ is not a $G_{\delta}$-subset of $S$.

\section{REFERENCES}

1. A. V. Arhangel'skiı̌, Behavior of metrizability under factor mapping, Dokl. Akad. Nauk SSSR 164 (1965), 247-250=Soviet Math. Dokl. 6 (1965), 1187-1190. MR 33 \#697.

2. - Mappings and spaces, Uspehi Mat. Nauk 21 (1966), no. 4 (130), 133-184= Russian Math. Surveys 21 (1966), no. 4, 115-162. MR 37 \#3534.

3. - Bicompact sets and the topology of spaces, Trudy Moskov. Mat. Obšč. 13 (1965), 3-55=Trans. Moscow Math. Soc. 1965, 1-62. MR 33 \#3251.

4. D. K. Burke, Subparacompact spaces, Proc. Washington State Univ. Conf. on General Topology (Pullman, Wash., 1970), Pi Mu Epsilon, Dept. of Math., Washington State University, Pullman, Wash., 1970, pp. 39-49. MR 42 \#1066.

5. D. K. Burke and R. A. Stoltenberg, Some properties of $\pi$-images of metric spaces (to appear). 
6. G. Hamel, Eine Basis aller Zahlen und die unstetigen Losungen der funktional gleichung: $f(x+y)=f(x)+f(y)$, Math. Ann. 60 (1905), 459-462.

7. R. W. Heath, Semi-metric and related spaces, Topology Conference, Arizona State University, Tempe, Ariz., 1967, pp. 153-161.

8. S. I. Nedev, Symmetrizable spaces and final compactness, Dokl. Akad. Nauk SSSR 175 (1967), 532-534=Soviet Math. Dokl. 8 (1967), 890-892. MR 35 \#7293.

9. J. Novák, Induktion partiell stetiger Funktionen, Math. Ann. 118 (1942), 449-461. MR 6, 164.

Department of Mathematics, Northern Arizona University, Flagstaff, ARIzona 86001 (Current address)

Department of Mathematics, Arizona State University, Tempe, Arizona 85281 